\title{
Étude de la production des ions bromate lors de l'ozonation des eaux de la Banlieue de Paris : choix du mode d'ozonation et variation des param tres physico-chimiques Study of the production of bromate during ozonation of Paris-area source waters: ozonation mode and variation of the physical and chemical parameters
}

\author{
K. Gelinet, J. P. Croue, C. Galey, A. Laplanche et B. Legube
}

Volume 11, numéro 4, 1998

URI : https://id.erudit.org/iderudit/705323ar

DOI : https://doi.org/10.7202/705323ar

\section{Aller au sommaire du numéro}

\section{Éditeur(s)}

Université du Québec - INRS-Eau, Terre et Environnement (INRS-ETE)

\section{ISSN}

0992-7158 (imprimé)

1718-8598 (numérique)

\section{Découvrir la revue}

Citer cet article

Gelinet, K., Croue, J. P., Galey, C., Laplanche, A. \& Legube, B. (1998). Étude de la production des ions bromate lors de l'ozonation des eaux de la Banlieue de Paris : choix du mode d'ozonation et variation des param tres physico-chimiques. Revue des sciences de l'eau / Journal of Water Science, 11(4), 599-516. https://doi.org/10.7202/705323ar

\section{Résumé de l'article}

Cette étude a permis d'évaluer l'importance de la concentration en ions bromure, de la température et de la nature de la Matière Organique Naturelle (MON) sur la production des ions bromate en s'appuyant sur des expériences conduites en laboratoire et sur pilote semi-industriel (Centre d'Essais de Méry-sur-Oise).

Trois campagnes d'ozonation effectuées en parallèle à Méry-sur-Oise et au LCEE (Laboratoire de Chimie de l'Eau et de l'Environnement) sur des eaux filtrées sable, ont montré que les expériences conduites en laboratoire et sur pilote semi-industriel mènent à des résultats similaires, soit une relation linéaire $\left[\mathrm{BrO}_{3}-\mathrm{]}=\mathrm{f}(\mathrm{C} \cdot \tau)\right.$ vérifiant une pente identique pour des conditions expérimentales données (teneur en ions bromure, température, origine de l'eau). Ces travaux ont montré de façon nouvelle qu'une faible variation de la concentration en ions bromure ( \pm 15 à $\left.20 \mu \mathrm{g} . \mathrm{L}^{-1}\right)$ suffisait à modifier significativement la formation des ions bromate. A C $\cdot \tau=10$ et $\mathrm{T}=21^{\circ} \mathrm{C}$, la production des ions bromate est passée de 16 à $27 \mu \mathrm{g} . \mathrm{L}^{-1}$ pour une augmentation de la concentration en ions bromure de 80 à $95 \mu \mathrm{g} . \mathrm{L}^{-1}$.

Les résultats obtenus ont montré de plus que la température est un facteur important puisqu'une différence de $8^{\circ} \mathrm{C}\left(13\right.$ à $\left.21^{\circ} \mathrm{C}\right)$ a entraîné, pour la même eau ( $80 \mu \mathrm{g} . \mathrm{L}^{-1}$ d'ions bromure, $C \cdot \tau=10$ ), une augmentation de la concentration en ions bromate de 10 à $16 \mu$ g.. ${ }^{-1}$. Pour d'autres eaux (Seine, Marne et Oise), trois autres campagnes conduites avec des eaux clarifiées ont été effectuées après ajustement de la teneur en ions bromure et régulation de la température, ces trois eaux présentant par ailleurs des caractéristiques similaires en ce qui concerne le $\mathrm{pH}$ et l'alcalinité.

A C. $\tau$ équivalent, la production d'ions bromate s'est avérée significativement plus faible pour l'eau de l'Oise que pour les deux autres eaux. La nature de la MON pourrait donc avoir une influence notable sur la formation des ions bromate.
Tous droits réservés @ C Revue des sciences de l'eau, 1998

Ce document est protégé par la loi sur le droit d'auteur. L'utilisation des services d'Érudit (y compris la reproduction) est assujettie à sa politique d'utilisation que vous pouvez consulter en ligne.

https://apropos.erudit.org/fr/usagers/politique-dutilisation/ 


\title{
Étude de la production des ions bromate lors de l'ozonation des eaux de la banlieue de Paris : choix du mode d'ozonation et variation des paramètres physicochimiques
}

\author{
Study of the production of bromate during ozonation \\ of Paris-area source waters: ozonation mode \\ and variation of the physical and chemical parameters
}

K. GELINET ${ }^{1}$ * , J.P. CROUÉ 1 , C. GALEY², A. LAPLANCHE ${ }^{3}$ et B. LEGUBE ${ }^{1}$

Reçu le 12 mai 1997, accepté le 24 avril 1999**.

SUMMARY

The publication of Kurokawa $e t$ al. in 1990 confirming the toxicity of bromate of rats and mice, initiated the research effort that was internationally conducted during the last seven years to better understand the reaction mechanisms of bromate formation during the ozonation of natural waters. Based on the research findings regarding the effect of a number of parameters (bromide, ozone dose, $\mathrm{pH}$, temperature, alkalinity, DOC content, ammonia...), predictive models (empirical and reaction kinetic based models), including molecular and/or radical pathways, have been developed with more or less success. Complementary results are still needed to better understand this complex mechanism.

The main objective of our work was to evaluate how the seasonal variation of the physical chemical characteristics of Paris-area source waters (i.e. bromide content, temperature, natural organic matter) can affect the production of bromate during ozonation.

In order to confirm that lab-scale experiments could be proposed to develop such research program, parallel tests were first conducted at the bench- and pilot-scale based on comparable $C \cdot \tau$ conditions. The lab-scale reactor was a $380 \mathrm{~mL}$ glass column (internal diameter: $0.02 \mathrm{~m}$; height: $1.2 \mathrm{~m}$ ) equipped with a water jacket to allow temperature to be varied and maintained. These reactor was used as a continuous flow reactor with recirculation. The pilot-scale ozonation contactor installed at the Méry sur Oise water treatment plant was

1. Laboratoire de Chimie de l'Eau et de l'Environnement (LCEE), UPRES A CNRS 6008, ESIP, 40, avenue du Recteur Pineau, 86000 Poitiers, France.

2. Compagnie Générale des Eaux (CGE), Service Equipement, Quartier Valmy, 32, place Ronde, 92982 ParisLa-Défense Cedex, France.

3. Laboratoire de Chimie des Nuisances et Génie de l'Environnement (LCNGE), ENSCR, avenue du Général Leclerc, 35000 Rennes Beaulieu, France.

* Correspondance.

* * Les commentaires seront reçus jusqu'au 31 juillet 1999. 
comprised of four 30-liter columns in series (diameter: $0.1 \mathrm{~m}$; height: $4 \mathrm{~m}$ ). The first column is used as the application column while the three others are used as residence column. The results have shown that lab-scale ozonation experiments conducted on Méry sur Oise sand filtered water led to similar results compared to pilot ozonation conducted on the same water and at the same temperature (sampled the same day) using the Méry sur Oise pilot-scale reactor. For applied $\mathrm{C} \cdot \tau$ that ranged from 4 to $20 \mathrm{mg} \mathrm{O} / \mathrm{L} \cdot \mathrm{min}$, similar linear relationship between bromate formation and applied $\mathcal{C} \cdot \tau$ was obtained with the two reactors.

A survey conducted on the Oise River has shown that the bromide concentration ranged from $40 \mu \mathrm{g} / \mathrm{L}$ (winter period) to $80 \mu \mathrm{g} / \mathrm{L}$ (summer period). If it is already well known that higher the bromide content, higher the bromate formation, our work has also pointed out that even a small increase of the bromide concentration from 80 to $95 \mu \mathrm{g} / \mathrm{L}(15 \mu \mathrm{g} / \mathrm{L}$ of bromide spiked as $\mathrm{KBr})$ can significantly impact the bromate formation (same experimental conditions) that, as an example, increased from 16 to $27 \mu \mathrm{g} / \mathrm{L}$ for $\mathrm{C} \cdot \tau$ of 10 at $21^{\circ} \mathrm{C}$.

The temperature of the Oise river can vary from $5^{\circ} \mathrm{C}$ up to $25^{\circ} \mathrm{C}$. Using carefully controlled temperature conditions, one can observed that the slope of the bromate production versus applied $\mathrm{C} \cdot \tau$ increased with increasing temperature (same water). For example, the production of bromate during the ozonation (applied $C \cdot \tau=10$ ) of the Méry sur Oise sand filtered water was 7, 10 and $16 \mu \mathrm{g} / \mathrm{L}$ for 5,13 and $21^{\circ} \mathrm{C}$, respectively. Complementary experiments, have shown that the impact of the variation of the initial bromide concentration was proportionally more important for low-temperature water $\left(5\right.$ to $\left.13^{\circ} \mathrm{C}\right)$ than for moderate-temperature water $\left(20^{\circ} \mathrm{C}\right)$.

The origin and nature of the water is considered to play a significant role on the formation of bromate during ozonation, however few studies have evaluated the importance of these parameters using carefully controlled experimental conditions. In order to better define how important is the change in bromate production with the modification of the quality of the Paris suburbs water sources, especially the organic content (nature and concentration of the NOM), two sets of experiments were conducted.

In the first part of the work, the Merry sur Oise sand filtered water was sampled at three different periods of the year 1996 (June, July and December), and the ozonation experiments were conducted at the same temperature $\left(21^{\circ} \mathrm{C}\right)$ after bromide concentration was adjust to $80 \mu \mathrm{g} / \mathrm{L}$. The three water samples had the same pH and did not contain ammonia. Significant differences were observed in the bromate production, showing a larger production with the winter water as compared to the summer water. The fact that the winter water was enriched in DOC (3.7 mg/L of DOC) as compared to the two others (2.6 $2.7 \mathrm{mg} / \mathrm{L}$ of DOC) may explain this difference since a larger ozone dose was probably necessary (ozone transfert not controlled because of the small size of the lab-scale reactor) to reach the same applied $C \cdot \tau$ due to a higher ozone consumption from the natural organic matter. The slightly lower alkalinity of the winter sample (200 mg/L as $\mathrm{CaCO}_{3}$ as compared to $250 \mathrm{mg} / \mathrm{L} \mathrm{CaCO}$ for the summer samples) could have led to a less pronounced scavenger effect, condition that favors the radical pathway which is generally predominant. However, it is also known that carbonate species can also promote the formation of bromate due to the production of carbonate radicals. Comparing the results obtained with the water samples collected during the summer period, more bromate was produced in July than in June. The higher hydrophobic character (more aromatic in character) of the NOM of the water sampled in July (SUVA $\mathbf{2 . 1 5}$ ) as compared to the June sample (SUVA $=1.88$ ), characteristic that favor the ozone consumption and consequently the $\mathrm{OH}$ radical production, may justify this finding. 
In the second part of the work, the bromate formation obtained during the ozonation of the three major water sources of the Paris suburbs (sampled after clarification), Oise River, Marne River and Seine River, was compared (same temperature) after the bromide content was adjust to $80 \mu \mathrm{g} / \mathrm{L}$. Similar results were obtained with the clarified Marne river and Seine River, the two waters showing the same physical chemical characteristics $(2.2$ and $2.5 \mathrm{mg} / \mathrm{L}$ of DOC; pH 7.9 and 7.8; Alkalinity: 225 and $210 \mathrm{mg} / \mathrm{L}$ as $\mathrm{CaCO}_{3}$ ). A lower production of bromate as a function of the applied $C \cdot \tau$ was observed with the clarified Oise river, result that is in contradiction with our previous hypotheses since this water source showed the highest DOC content, the highest SUVA and the lowest alkalinity among the three waters studied.

More work needs to be done to better understand the impact of the origin and nature of the NOM on the bromate formation mechanisms. As a general conclusion, this work also confirmed that the physical chemical characteristics of source water (DOC, temperature, alkalinity, bromide content...) are more important factors as compared to the hydraulic characteristics of the reactor.

Key-words: ozonation, bromate ions, $C \cdot \tau$, bromide ions, temperature.

Cette étude a permis d'évaluer l'importance de la concentration en ions bromure, de la température et de la nature de la matière organique naturelle (MON) sur la production des ions bromate en s'appuyant sur des expériences conduites en laboratoire et sur pilote semi-industriel (Centre d'essais de Mérysur-Oise).

Trois campagnes d'ozonation effectuées en parallèle à Méry-sur-Oise et au LCEE (Laboratoire de Chimie de l'Eau et de l'Environnement) sur des eaux filtrées sable, ont montré que les expériences conduites en laboratoire et sur pilote semi-industriel mènent à des résultats similaires, soit une relation linéaire $\left[\mathrm{BrO}_{3}-\mathrm{f}=\mathrm{f}(\mathrm{C} \tau)\right.$ vérifiant une pente identique pour des conditions expérimentales données (teneur en ions bromure, température, origine de l'eau).

Ces travaux ont montré de façon nouvelle qu'une faible variation de la concentration en ions bromure ( \pm 15 à $\left.20 \mu \mathrm{g} \cdot \mathrm{L}^{-1}\right)$ suffisait à modifier significativement la formation des ions bromate. $\ddot{\mathrm{A}} \mathrm{C} \tau=10$ et $\mathrm{T}=21^{\circ} \mathrm{C}$, la production des ions bromate est passée de 16 à $27 \mu \mathrm{g} \cdot \mathrm{L}^{-1}$ pour une augmentation de la concentration en ions bromure de 80 à $95 \mu \mathrm{g} \cdot \mathrm{L}^{-1}$.

Les résultats obtenus ont montré de plus que la température est un facteur important puisqu'une différence de $8{ }^{\circ} \mathrm{C}\left(13\right.$ à $\left.21^{\circ} \mathrm{C}\right)$ a entrâné, pour la même eau $\left(80 \mu \mathrm{g} \cdot \mathrm{L}^{-1}\right.$ d'ions bromure, $\left.C \cdot \tau=10\right)$, une augmentation de la concentration en ions bromate de 10 à $16 \mu \mathrm{g} \cdot \mathrm{L}^{-1}$.

Pour d'autres eaux (Seine, Marne et Oise), trois autres campagnes conduites avec des eaux clarifiées ont été effectuées après ajustement de la teneur en ions bromure et régulation de la température, ces trois eaux présentant par ailleurs des caractéristiques similaires en ce qui concerne le pH et l'alcalinité.

À C- $\tau$ équivalent, la production d'ions bromate s'est avérée significativement plus faible pour l'eau de l'Oise que pour les deux autres eaux. La nature de la MON pourrait donc avoir une influence notable sur la formation des ions bromate.

Mots clés : ozonation, ions bromate, $C \cdot \tau$, ions bromure, température. 


\section{1 - INTRODUCTION}

Suite au projet de législation concernant la concentration maximale admise des ions bromate dans les eaux de consommation (une proposition de Directive Européenne (131/5 du 30/05/95) a en effet récemment été proposée à $10 \mu \mathrm{g} \cdot \mathrm{L}^{-1}$ alors que la recommandation de l'Organisation Mondiale de la Santé (OMS) est de $\left.25 \mu \mathrm{g} \cdot \mathrm{L}^{-1}\right)$, l'étude des conditions de formation des ions bromate dans les filières de production d'eau potable utilisant l'ozone, est d'actualité. Malgré les nombreux travaux de recherche publiés dans ce domaine (AMY et al., 1995 ; LEGUBE et al., 1995 ; ROUSTAN et al., 1996 ; SONG et al., 1996 ; VON GUNTEN and HOIGNÉ, 1994) et en dépit de la qualité des résultats obtenus, le développement d'un modèle prédictif adapté aux eaux naturelles, reste encore aujourd'hui un enjeu économique. Plusieurs questions restent donc posées, notamment sur la transposition aux usines de production des essais menés à l'échelle du laboratoire, ou encore sur l'impact de la qualité des eaux sur la formation des ions bromate.

Afin d'approfondir ces quelques points, une étude consistant à évaluer l'importance de la concentration en ions bromure, de la température et de l'origine de l'eau sur la production des ions bromate s'est appuyée sur des expériences conduites en laboratoire et sur le pilote de taille semi-industrielle du Centre d'essais de Méry-sur-Oise (CGE).

\section{2 - MATÉRIEL ET MÉTHODES}

\subsection{Caractéristiques des eaux étudiées}

L'étude a été conduite à partir d'eaux filtrées sable (EFS) prélevées sur les usines de Méry-sur-Oise, Neuilly-sur-Marne et Choisy-le-Roi. La qualité physicochimique de ces eaux prélevées entre mai et novembre 1996 a sensiblement évolué comme le montre le tableau 1.

Quelles que soient les campagnes d'étude menées, la présence d'ions bromate dans les eaux avant ozonation n'a jamais été observée (limite de détection de $2 \mu \mathrm{g} \cdot \mathrm{L}^{-1}$ de $\mathrm{BrO}_{3}-$ ).

\subsection{Les réacteurs}

Les essais ont été réalisés sur le pilote semi-industriel de Méry-sur-Oise et en laboratoire pour les eaux de Méry-sur-Oise, et uniquement en laboratoire pour les eaux de Neuilly-sur-Marne et Choisy-le-Roi.

\subsubsection{Pilote semi-industriel}

Le pilote semi-industriel du Centre d'Essais de Méry-sur-Oise (figure 1), est constitué d'une colonne d'ozonation (colonne $n^{\circ} 1$ ) équipée de quatre points de prélèvement et de trois colonnes de contact de 30 litres chacune (diamètre: $0,1 \mathrm{~m}$ - hauteur moyenne : $4 \mathrm{~m}$ ). Pour les trois autres colonnes, seul le prélèvement en sortie peut être effectué. 
Tableau 1 Caractéristiques des différentes eaux étudiées.

Table 1 Characteristics of the studied waters.

\begin{tabular}{|c|c|c|c|c|c|c|c|c|c|c|}
\hline \multirow{2}{*}{$\begin{array}{l}\text { Origine } \\
\text { des } \\
\text { eaux }\end{array}$} & \multirow{2}{*}{$\begin{array}{c}\mathbf{N}^{\circ} \\
\text { camp. }\end{array}$} & \multirow{2}{*}{ Date } & $\begin{array}{c}\text { Temp. } \\
\left({ }^{\circ} \mathrm{C}\right)\end{array}$ & $\begin{array}{c}\mathrm{BrO}_{\mathrm{J}}^{-} \\
\left(\mu \mathrm{g} \cdot \mathrm{L}^{-1}\right)\end{array}$ & $\left(\begin{array}{c}B r \\
\left(\mu g \cdot L^{-3}\right)\end{array}\right)$ & $\mathrm{pH}$ & \multirow{2}{*}{\begin{tabular}{|c|}
$\begin{array}{c}\text { Alcalinité } \\
\text { (méq. } L^{-1} \text { ) }\end{array}$ \\
1 \\
\end{tabular}} & \multirow{2}{*}{\begin{tabular}{|c|}
$\begin{array}{c}\mathrm{COD} \\
\left(\mathrm{mg} \mathrm{C} \cdot \mathrm{L}^{-1}\right)\end{array}$ \\
2 \\
\end{tabular}} & \multirow{2}{*}{\begin{tabular}{|c|}
$\begin{array}{c}N-\mathrm{NH}_{4^{+}} \\
\left(\mathrm{mg} \cdot \mathrm{L}^{-1}\right)\end{array}$ \\
1 \\
\end{tabular}} & \multirow{2}{*}{$\begin{array}{c}\text { UV } 254 \\
(4 \mathrm{~cm})\end{array}$} \\
\hline & & & 1 & 2 & 2 & 12 & & & & \\
\hline $\begin{array}{c}\text { Méry- } \\
\text { sur-Oise }\end{array}$ & $\begin{array}{l}1 \\
2 \\
3 \\
4 \\
5 \\
6\end{array}$ & $\begin{array}{l}20.05 .96 \\
26.06 .96 \\
16.07 .96 \\
22.10 .96 \\
04.11 .96 \\
02.12 .96\end{array}$ & $\begin{array}{r}13,0 \\
21,0 \\
20,0 \\
14,0 \\
12,6 \\
6,0\end{array}$ & $\begin{array}{l}<2 \\
<2 \\
<2 \\
<2 \\
<2 \\
<2\end{array}$ & $\begin{array}{l}60 \\
80 \\
80 \\
50 \\
45 \\
35\end{array}$ & $\begin{array}{l}7,4-7,8 \\
7,4-7,6 \\
7,4-7,6 \\
7,4-7,5 \\
7,5-7,4 \\
7,7-7,6\end{array}$ & $\begin{array}{l}4,7 \\
4,6 \\
5,0 \\
4,9 \\
5,1 \\
3,9\end{array}$ & $\begin{array}{l}2,4 \\
2,6 \\
2,7 \\
2,6 \\
2,8 \\
3,7\end{array}$ & $\begin{array}{r}0,02 \\
<0,01 \\
<0,01 \\
0,01 \\
0,01 \\
<0,01\end{array}$ & $\begin{array}{l}0,182 \\
0,196 \\
0,232 \\
0,231 \\
0,264 \\
0,340\end{array}$ \\
\hline $\begin{array}{l}\text { Neuilly- } \\
\text { sur- } \\
\text { Marne }\end{array}$ & $\begin{array}{l}4 \\
5 \\
6\end{array}$ & $\begin{array}{l}22.10 .96 \\
04.11 .96 \\
28.11 .96\end{array}$ & $\begin{array}{r}13,4 \\
11,9 \\
5,5\end{array}$ & $\begin{array}{l}<2 \\
<2 \\
<2\end{array}$ & $\begin{array}{l}30 \\
25 \\
20\end{array}$ & $\begin{array}{l}7,7-7,8 \\
7,8-7,7 \\
7,9-7,8\end{array}$ & $\begin{array}{l}3,6 \\
3,6 \\
4,5\end{array}$ & $\begin{array}{l}1,5 \\
1,8 \\
2,2\end{array}$ & $\begin{array}{r}0,03 \\
0,01 \\
<0,01\end{array}$ & $\begin{array}{l}0,112 \\
0,120 \\
0,168\end{array}$ \\
\hline $\begin{array}{l}\text { Choisy- } \\
\text { le-Roi }\end{array}$ & $\begin{array}{l}4 \\
5 \\
6\end{array}$ & $\begin{array}{l}22.10 .96 \\
04.11 .96 \\
28.11 .96\end{array}$ & $\begin{array}{r}13,2 \\
12,1 \\
5,6\end{array}$ & $\begin{array}{l}<2 \\
<2 \\
<2\end{array}$ & $\begin{array}{l}30 \\
25 \\
30\end{array}$ & $\begin{array}{l}7,6-7,5 \\
7,6-7,5 \\
7,8-7,7\end{array}$ & $\begin{array}{l}3,3 \\
3,4 \\
4,2\end{array}$ & $\begin{array}{l}1,6 \\
1,5 \\
2,5\end{array}$ & $\begin{array}{r}0,02 \\
0,01 \\
<0,01\end{array}$ & $\begin{array}{l}0,092 \\
0,084 \\
0,164\end{array}$ \\
\hline
\end{tabular}

1: Analyse réalisée sur le terrain le jour du prélèvement.

2: Analyse réalisée au laboratoire de Poitiers.

Le pilote est alimenté en continu par les eaux filtrées sable de l'usine d'eau potable de Méry-sur-Oise, le débit d'alimentation variant selon les besoins de 300 à $500 \mathrm{~L} \cdot \mathrm{h}^{-1}$. L'ozonation est effectuée à contre-courant dans la colonne $\mathrm{n}^{\circ} 1$ à des taux de traitement variants de 1,0 à $2,5 \mathrm{mg} \cdot \mathrm{L}^{-1}$ en ozone appliqué. Le comportement hydraulique de ce pilote est assez proche d'un flux piston puisque :

$$
\tau_{10}=0,7 \times \text { temps de séjour hydraulique }
$$

avec temps de séjour hydraulique $=\frac{\text { volume du réacteur }}{\text { débit moyen d'alimentation }}$

\subsubsection{Pilote de laboratoire}

Le pilote utilisé (figure 1) est un réacteur double enceinte de $380 \mathrm{~mL}$ parfaitement agité (diamètre interne : $0,02 \mathrm{~m}$-hauteur : 1,2 m). Le débit de recirculation qui est au moins égal à cinq fois le débit d'alimentation, a pour but d'homogénéiser le milieu réactionnel. Les ozonations ont été effectuées à co-courant et les taux d'ozone appliqués ont varié de 1,0 à $2,0 \mathrm{mg} \cdot \mathrm{L}^{-1}$.

\subsubsection{Méthodes analytiques}

La concentration en ozone dissous a été déterminée par la méthode spectrophotométrique au carmin indigo trisulfonate de potassium à $600 \mathrm{~nm}$ (BADER and HOIGNE, 1981).

Les dosages des ions bromate (colonne AS9-SC) et des ions bromure (colonne AS 12A) ont été réalisés par chromatographie ionique couplée à une détection conductimétrique. La précision du dosage des ions bromate est de $\pm 10 \%$, la limite de détection étant de $2 \mu \mathrm{g} \cdot \mathrm{L}^{-1}$ (KOUDJONOU et al., 1995) et celle du dosage des ions bromure est de $\pm 15 \%$, la limite de détection étant de $20 \mu \mathrm{g} \cdot \mathrm{L}^{-1}$ (norme ISO 10304-1:1992).

Le dosage de l'alcalinité de l'eau a été effectué par la méthode potentiométrique de la norme NF T90-036 (Afnor 1994). 
a)

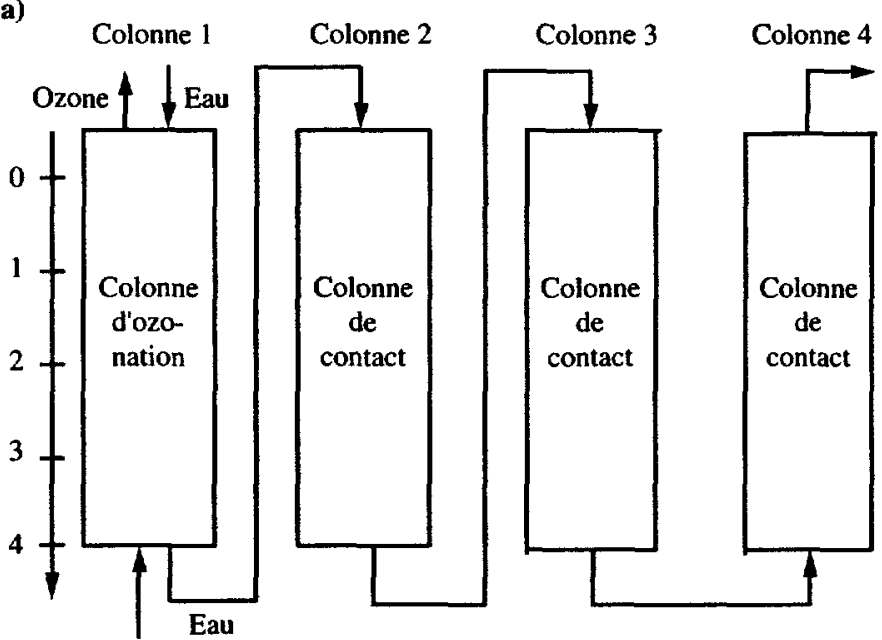

Ozone

b)

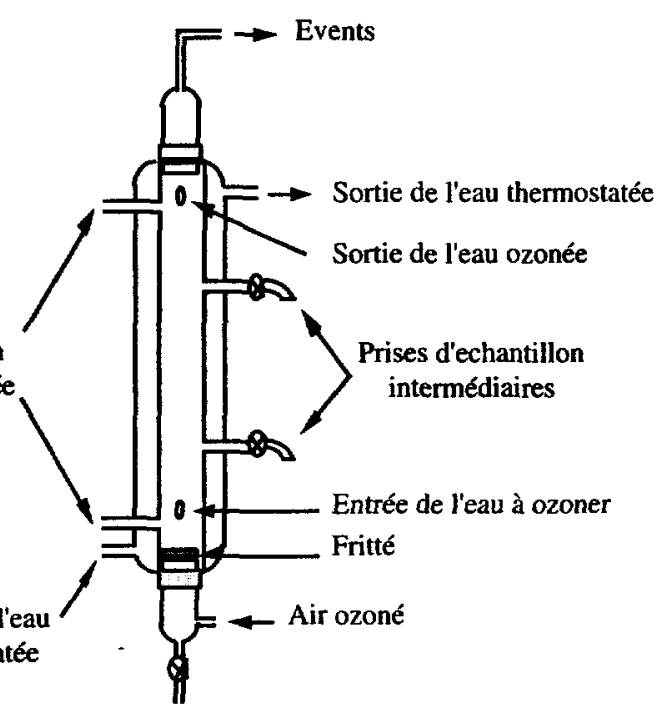

Figure 1 Schéma du pilote de Méry-sur-Oise (a) et de laboratoire (b).

Méry-sur-Oise pilot-scale ozonation unit (a) and lab-scale ozonation reactor (b).

L'analyse du carbone organique dissous (COD) des échantillons a été effectuée à l'aide d'un analyseur de carbone DOHRMANN DC-80 par dosage infrarouge du $\mathrm{CO}_{2}$ issu de l'oxydation UV-persulfate des composés organiques dissous.

Le dosage de l'azote ammoniacal a été effectué par la méthode par spectrométrie d'absorption moléculaire de la norme NF T90-015 (Afnor 1994).

L'absorbance à $254 \mathrm{~nm}$ des échantillons bruts a été mesurée sur un spectrophotomètre SAFAS dans des cuves en quartz de trajet optique $4 \mathrm{~cm}$. 


\subsubsection{Détermination du C. $\tau$}

\subsubsection{Pilote semi-industriel}

Pour le pilote de Méry-sur-Oise, la mesure du temps de séjour hydraulique « $\tau$ » de l'eau dans le réacteur se calcule de la façon suivante :

$$
\tau=\frac{\text { volume du réacteur }}{\text { débit d'alimentation }}
$$

La concentration moyenne en ozone dissous au sein de la colonne d'ozonation (colonne $n^{\circ}$ ) a été déterminée par l'intégration de la courbe « $C=f(\tau)$ » obtenue ( $\tau$ étant fonction du point de prélèvement) grâce aux différents prélèvements effectués sur cette colonne (figure 1).

Pour un prélèvement effectué en sortie de la colonne $\mathrm{i}$, le «C. $\tau$ total » a été calculé de la façon suivante:

$$
C \cdot \tau=\Sigma C_{i} \text { moyen } \times \tau_{i}
$$

Avec : $\mathrm{C} \cdot \tau$ en $\mathrm{mg} \mathrm{O}_{3} \mathrm{~L}^{-1} \cdot \mathrm{min}$.

$\mathrm{C}_{\mathrm{i}}$ moyen $=\left(\left(\mathrm{C}_{\text {entrée colonne } \mathrm{i}}+\mathrm{C}_{\text {sortie colonne } \mathrm{j}}\right) / 2\right)$ en $\mathrm{mg} \mathrm{O}_{3} \mathrm{~L}^{-1}$.

$t_{i}$ : temps hydraulique en min (volume colonne i/débit).

\subsubsection{Pilote de laboratoire}

Le pilote de laboratoire étant un réacteur parfaitement agité, la concentration en ozone dissous " $\mathrm{C}$ " reste constante dans toute la colonne, ce qui a été plusieurs fois vérifié par des mesures en milieu et sortie de colonne. La mesure du temps de séjour " $\tau$ » de l'eau dans le réacteur se calcule comme pour le pilote de Méry-sur-Oise.

\section{3 - RÉSULTATS ET DISCUSSION}

\subsection{Choix du mode d'ozonation : comparaison du pilote semi-industriel et du module de laboratoire}

La commodité qu'offre la conduite des expériences à l'échelle du laboratoire est toujours appréciée et rend plus facile l'étude de l'influence des paramètres expérimentaux. La question de la transposition des données obtenues à l'échelle du laboratoire à des modules d'essais de taille semi-industrielle voire aux conditions réelles reste toujours posée.

Une étude comparative entre pilote de laboratoire et pilote semi-industriel a été effectuée sur les eaux filtrées sable de Méry-sur-Oise.

Pour tous les essais effectués, les résultats obtenus ont mis nettement en évidence une relation linéaire entre la concentration en ions bromate et le C. $\tau$ appliqué. Plus le $\mathrm{C} \cdot \tau$ augmente, plus la teneur en ions bromate en solution croît. $\mathrm{Ce}$ type de relation a déjà été établi par d'autres auteurs sur des eaux d'origine diverse (KRASNER et al., 1993a; KRUITHOF et al., 1995 ; VON GUNTEN and HOIGNÉ, 1994). 
La figure 2 ( $a$ et $b$ ) présente les résultats obtenus sur l'eau filtrée sable (EFS) de Méry-sur-Oise lors de la campagne 2 sans ou avec dopage en ions bromure $\left(+30 \mu \mathrm{g} \cdot \mathrm{L}^{-1} \mathrm{Br}\right)$.

Pour un C. $\tau$ appliqué équivalent, les résultats obtenus sur le pilote de laboratoire sont sensiblement identiques à ceux issus du pilote de Méry-sur-Oise. Les différences relevées restent en général minimes, et de l'ordre de grandeur de l'erreur analytique pour les très faibles $C \cdot \tau$ (cette observation a été également vérifiée lors de deux autres campagnes réalisées pour des températures et des concentrations en ions bromure différentes).

À la vue de ces résultats, tout indique que le pilote de laboratoire est bien adapté pour simuler un pilote de taille semi-industrielle comme celui de Méry-surOise.

Ces résultats sont en accord avec les travaux récents de AMY et al. (1997) et VON GUNTEN and HOIGNÉ (1994) qui ont montré qu'un réacteur semi-continu donnait des résultats comparables à ceux d'un réacteur de taille semi-industrielle, et que l'hydraulique du contacteur avait peu d'effet sur l'avancement de la réaction de l'ozone sur les ions bromures (ROUSTAN et al., 1996).

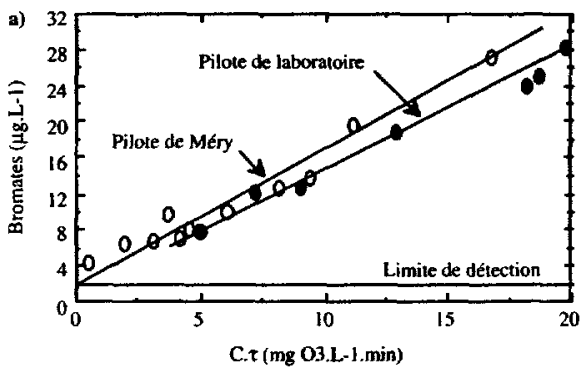

Campagne $n^{\circ} 2(26.06 .96)$ $21^{\circ} \mathrm{C}-80 \mu \mathrm{g} . \mathrm{L}^{-1} \mathrm{Br}-$

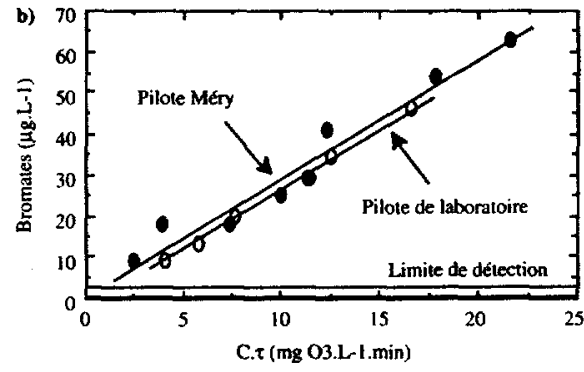

Campagne $n^{\circ} 2(26.06 .96)$ $21^{\circ} \mathrm{C}-110 \mu \mathrm{g} . \mathrm{L}^{-1} \mathrm{Br}$

Figure 2a, 2b Production d'ions bromate lors de l'ozonation de l'EFS de Méry-sur-Oise : étude comparative du pilote semi-industriel et du module de laboratoire.

Bromate formation during the ozonation of the sand-filtered water produced at the Méry-sur-Oise plant: Comparison of the results obtained with pilotscale and lab-scale experimental conditions.

\subsection{Les ions bromure}

L'étude des ions bromure est primordiale puisque ce sont les précurseurs des ions bromate. Ils sont naturellement présents dans les eaux de rivières à une concentration qui fluctue au cours du temps.

\subsubsection{Variation saisonnière des teneurs en ions bromure}

Le suivi analytique réalisé sur l'eau de Méry-sur-Oise (figure 3) montre que la teneur en ions bromure passe par un maximum pendant la période estivale avec une concentration de l'ordre de 70 à $80 \mu \mathrm{g} \cdot \mathrm{L}^{-1}$. Les valeurs relevées au printemps et en automne sont significativement plus faibles, de l'ordre de $45 \mu \mathrm{g} \cdot \mathrm{L}^{-1}$ à $50 \mu \mathrm{g} \cdot \mathrm{L}^{-1}$. 
Une telle évolution a été également observée en fonction de la saison aux USA (AMY, communication personnelle).

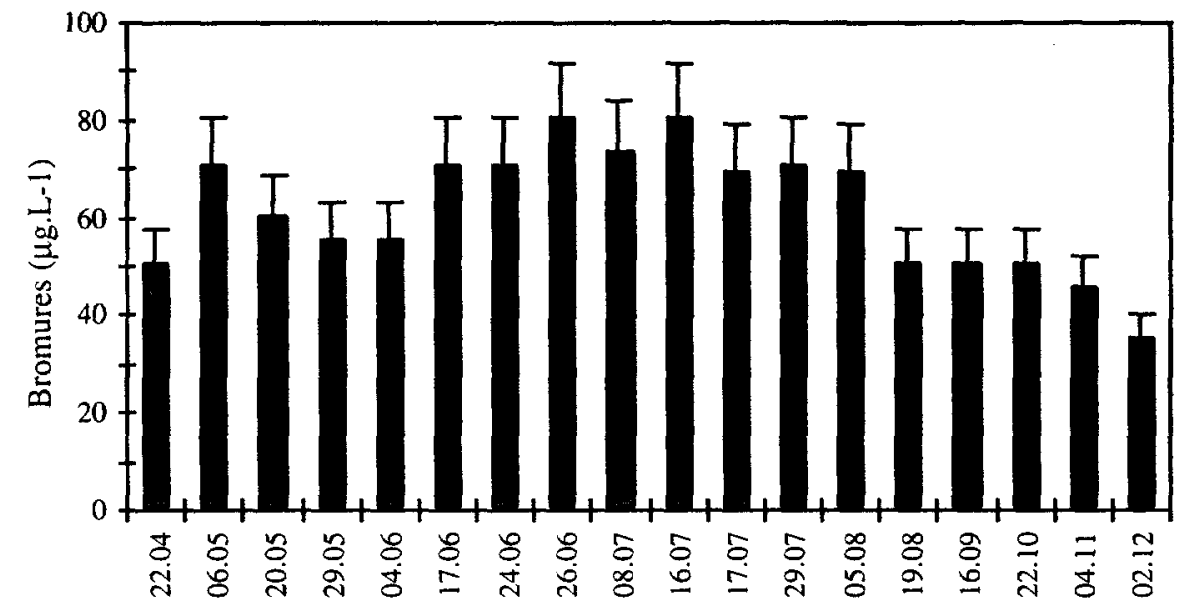

Dates des prélèvements

Figure 3 Relevé des concentrations en ions bromure sur l'eau étudiée de Méry-surOise au cours de l'année 1996.

Survey of bromide ion concentrations in the Oise River (year 1996).

\subsubsection{Relation entre la teneur initiale en ions bromure et la production des ions bromate}

\subsubsection{Cas d'une eau dopée en ions bromure}

L'impact de la concentration initiale en ions bromure a été étudiée en laboratoire lors des campagnes 2 et 3. Les eaux prélevées sur le site ont été dopées en ions bromure par ajout de bromure de potassium. Seuls les résultats de la campagne 3 sont présentés sur la figure 4 .

Quelle que soit la concentration en ions bromure après dopage, la production d'ions bromate est toujours proportionnelle au $\mathrm{C} \cdot \tau$. En revanche, la pente de la droite augmente avec la concentration en ions bromure.

Pour une température identique $\left(21^{\circ} \mathrm{C}\right.$ - campagne 3$)$, une augmentation de la teneur en ions bromure, même très faible $\left(+15 \mu \mathrm{g} \cdot \mathrm{L}^{-1}\right.$ obtenue par dopage à partir d'une solution concentrée de bromure de potassium $\mathrm{KBr}$ ), entraîne une augmentation non négligeable sur la pente de la droite. Pour un $\mathrm{C} \cdot \tau$ de 10 , la production d'ions bromate a atteint 17,27 et $35 \mu \mathrm{g} \cdot \mathrm{L}^{-1}$ pour des teneurs en bromure respectivement égales à 80,95 et $110 \mu \mathrm{g} \cdot \mathrm{L}^{-1}$.

De même, lors de la campagne 2, pour un $\mathrm{C} \cdot \tau$ de 10 et une température de $21^{\circ} \mathrm{C}$, la concentration en ions bromate a pratiquement doublé entre 80 et $110 \mu \mathrm{g} \cdot \mathrm{L}^{-1}$ d'ions bromure soit respectivement 14 et $28 \mu \mathrm{g} \cdot \mathrm{L}^{-1}$ d'ions bromate. 


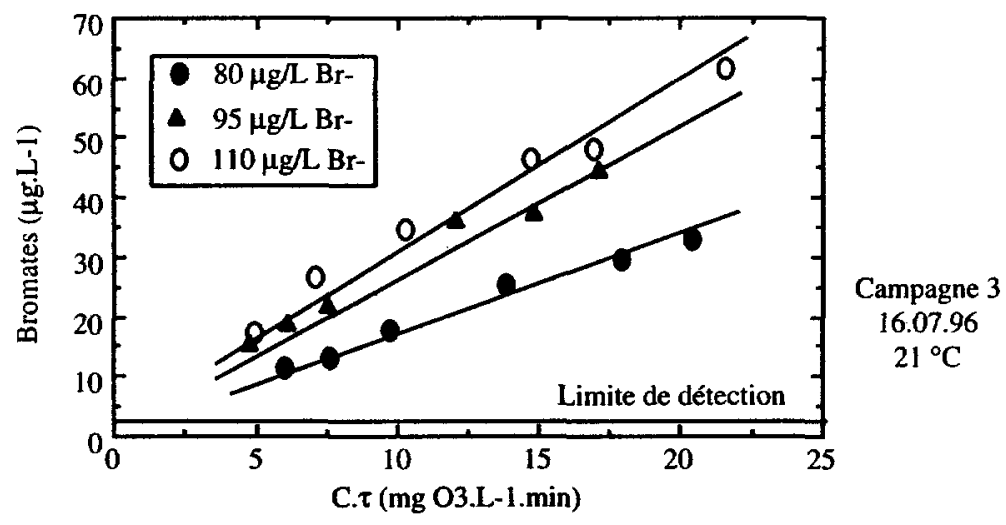

Figure 4 Étude de l'ozonation en laboratoire de l'EFS de Méry-sur-Oise : influence de la concentration en ions bromure.

Influence of the bromide ion content (spiked water) on the production of bromate by ozonation of the Méry-sur-Oise sand-filtered water (lab-scale reactor).

\subsubsection{Cas d'eaux de même origine mais prélevées à différentes époques}

Afin d'évaluer l'importance des variations saisonnières pour l'eau de Mérysur-Oise (avec ou sans dopage en ions bromure), huit corrélations $\left[\mathrm{BrO}_{3}-\right]$ $=\mathrm{f}(\mathrm{C} \cdot \tau)$ ont au total été établies en laboratoire pendant la période d'étude (mai à novembre 1996) à la température de $21^{\circ} \mathrm{C}$.

À partir de l'ensemble des données obtenues, la figure 5 présente l'évolution de la teneur en ions bromate en fonction de la concentration en ions bromure pour deux C. $\tau$ différents.

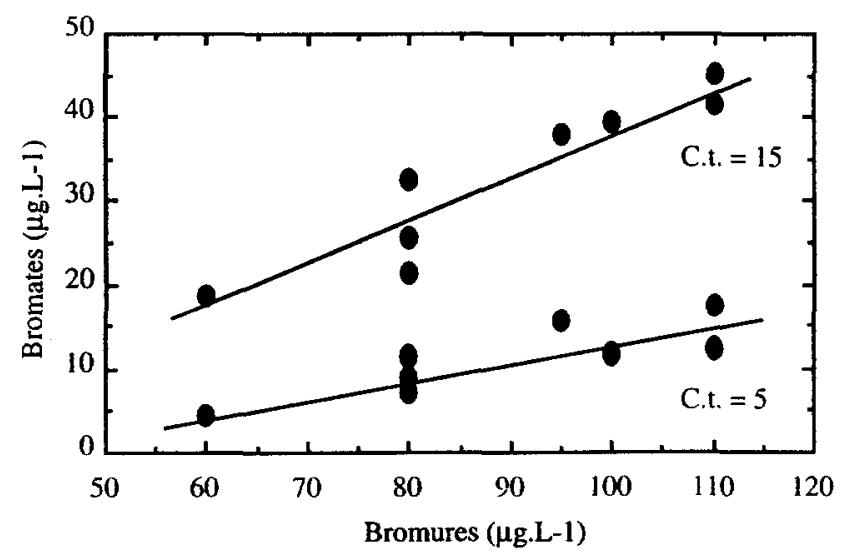

Figure 5 Étude en laboratoire de l'ozonation de l'EFS de Méry-sur-Oise à $21^{\circ} \mathrm{C}:$ formation d'ions bromate en fonction de la teneur en ions bromure à $\mathrm{C} \cdot \tau$ constant.

Bromate formation versus bromide content at constant $C \cdot \tau$ (Méry-sur-Oise sand-filtered water $-21^{\circ} \mathrm{C}-$ lab-scale reactor). 
Globalement, et pour les deux C - $\tau$ choisis, plus la concentration en ions bromure est élevée, plus la formation en ions bromate est importante. Le phénomène est d'autant plus net que le C. $\tau$ est fort.

Dans le détail, on relève néanmoins qu'à concentration en ions bromure équivalente soit $80 \mu \mathrm{g} \cdot \mathrm{L}^{-1}$ et à la même température $\left(21^{\circ} \mathrm{C}\right)$, la production d'ions bromate lors de l'ozonation de l'eau filtrée sable de Méry-sur-Oise présente des variations significatives en fonction de la période de prélèvement (20 à $30 \mu \mathrm{g} \cdot \mathrm{L}^{-1}$ d'ions bromate pour un $\mathrm{C} \cdot \tau$ de $15 \mathrm{mg} \mathrm{O} \mathrm{O}_{3} \cdot \mathrm{L}^{-1} \cdot \mathrm{min}$ ).

Cette constatation nous amène à penser qu'il existe d'autres paramètres influençant la production des ions bromate, ce que nous envisagerons par la suite.

\subsection{La température}

Pour les deux premières campagnes de prélèvement (eau filtrée sable de Méry-sur-Oise), nous avons étudié en laboratoire l'impact de la température de leau sur la production d'ions bromate par ozonation. La température a été fixée successivement à $5^{\circ} \mathrm{C}, 13^{\circ} \mathrm{C}$ puis $20-21^{\circ} \mathrm{C}$. Les résultats de la campagne 2 sont présentés sur la figure 6 .

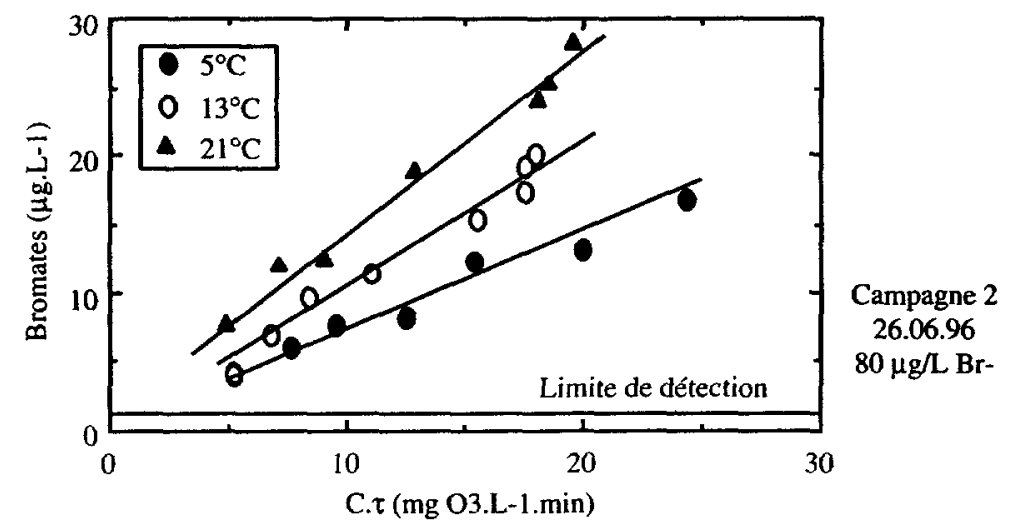

Figure 6 Étude en laboratoire de l'influence de la température sur la formation des ions bromate lors de l'ozonation de l'eau de Méry-sur-Oise.

Bromate formation as a function of C. $\tau$ : Effect of temperature (Méry-surOise sand filtered water - lab-scale reactor).

Quelle que soit la température de l'eau étudiée, on note que la formation d'ions bromate reste proportionnelle au $C \cdot \tau$. Toutefois, la température a une incidence sur la pente de cette droite qui augmente avec ce paramètre. Pour un $C \cdot \tau$ donné, la production d'ions bromate augmente donc avec la température, l'effet de ce paramètre étant d'autant plus marqué que le $C \cdot \tau$ est grand.

La température apparaît donc comme un paramètre majeur. Par exemple, pour un $C \cdot \tau$ de 10 , une eau dont la concentration en ions bromate est inférieure à $10 \mu \mathrm{g} \cdot \mathrm{L}^{-1}$ à 5 ou $13^{\circ} \mathrm{C}$ (hiver ou automne), dépassera ce niveau critique (proposition de directive européenne) pour une température estivale de $21^{\circ} \mathrm{C}$ dans les 
mêmes conditions de traitement et pour des caractéristiques physico-chimiques similaires.

En examinant les résultats des campagnes 1 et 2 (réactions d'ozonation conduites en laboratoire à différentes températures, figure 7 , il semble que l'importance de la concentration en ions bromure sur la formation d'ions bromate soit proportionnellement plus importante à faible température $\left(5\right.$ et $\left.13^{\circ} \mathrm{C}\right)$ qu'à température élevée $\left(20\right.$ et $\left.21^{\circ} \mathrm{C}\right)$.

En effet pour un C. $\tau$ de 10 et pour des concentrations respectives en ions bromure de 60 à $80 \mu \mathrm{g} \cdot \mathrm{L}^{-1}$, la production en ions bromate est la suivante :

- à $5^{\circ} \mathrm{C}$ elle passe de 3 à $7 \mu \mathrm{g} \cdot \mathrm{L}^{-1}$ (rapport $=2,3$ ) et à $13^{\circ} \mathrm{C}$ elle passe de 4 à $10 \mu \mathrm{g} \cdot \mathrm{L}^{-1}$ (rapport $=2,5$ ) ;

- à 20-21 ${ }^{\circ} \mathrm{C}$ elle passe de 11 à $15 \mu \mathrm{g} \cdot \mathrm{L}^{-1}$ (rapport $=1,4$ ).
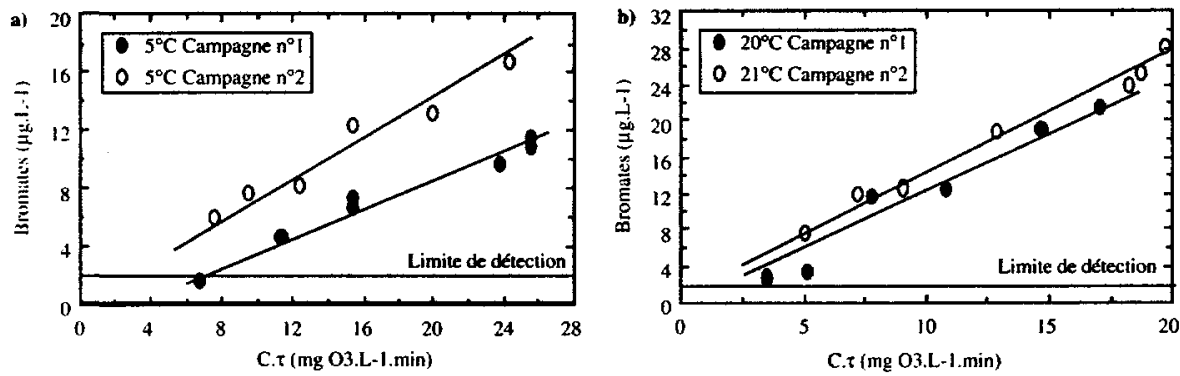

Figure 7 Importance de la teneur initiale en ions bromure sur la formation des ions bromate : influence de la température - EFS de Méry-sur-Oise - Réacteur de laboratoire.

Bromate formation versus applied C. $\tau$ : Importance of the temperature on the bromide content effect. (Méry-sur-Oise sand-filtered water, lab-scale reactor).

Pour une période donnée, la fluctuation accidentelle (ex.: pollution industrielle) de la teneur en ions bromure induira donc une augmentation en ions bromate proportionnellement plus importante en période froide qu'en période chaude.

Cependant, si on considère les valeurs absolues obtenues, il s'avère que la production des ions bromate est plus forte en été, d'une part en raison de la température plus élevée mais d'autre part en raison de la plus forte teneur des eaux en ions bromure à cette époque de l'année.

\subsection{Origine de l'eau}

Comme nous l'avons souligné précédemment, la teneur initiale en ions bromure ainsi que la température sont des paramètres très importants influençant la production des ions bromate.

Ce paragraphe, qui s'intéresse à l'origine de l'eau, ne prend pas en compte ces derniers puisqu'ils ont fait au préalable lobjet d'un réajustement (certains paramètres comme l'alcalinité ont cependant pu varier très légèrement). 
Deux approches ont été menées pour évaluer l'impact de l'origine (saison et lieu) de l'eau :

- Étude sur des eaux provenant de la même station à des périodes différentes et ozonées à même température en présence d'une teneur initiale en ions bromure identique.

- Étude sur des eaux prélevées le même jour sur les différents sites de Mérysur-Oise, Neuilly-sur-Marne et Choisy-le-Roi, l'ozonation étant conduite à la même température après réajustement de la teneur en ions bromure pour que celle-ci soit identique pour les trois eaux.

La température retenue a été de $21^{\circ} \mathrm{C}$ afin d'obtenir une forte production en ions bromate et de maximiser ainsi les effets éventuels de l'origine de l'eau.

Compte tenu des valeurs données dans le tableau 1, on considérera par la suite que les effets de l'azote ammoniacal et du pH sont négligeables.

\subsection{1 Étude des eaux issues d'un même site}

La figure 8 présente les résultats obtenus sur l'eau filtrée sable de Méry-surOise prélevée entre juin et début décembre 1996.

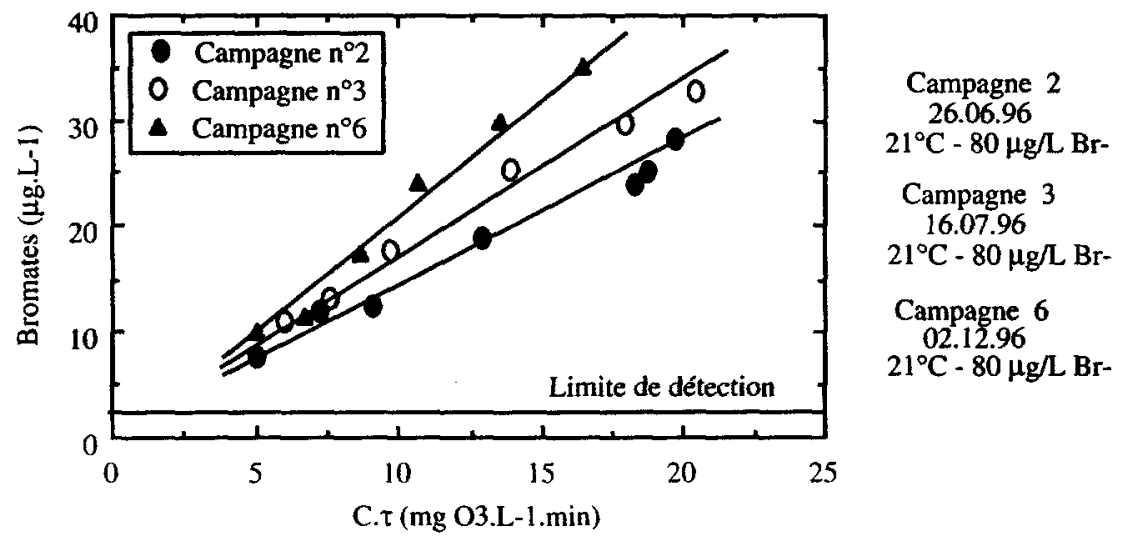

Figure 8 Étude en laboratoire de la formation des ions bromate lors de l'ozonation de I'EFS de Méry-sur-Oise à température et teneur en ions bromure constantes : variation saisonnière.

Seasonal variation of bromate formation at constant temperature and similar initial bromide content (Méry-sur-Oise sand-filtered water, lab-scale reactor).

On note que pour une température $\left(21^{\circ} \mathrm{C}\right)$ et une teneur en ions bromure $\left(80 \mu \mathrm{g} \cdot \mathrm{L}^{-1}\right)$ identiques, la formation des ions bromate varie en fonction de la période de prélèvement. En considérant que l'erreur sur la détermination de la teneur en ions bromure dans les eaux n'a qu'une incidence limitée sur la production des ions bromate à $21^{\circ} \mathrm{C}$ (figure 8 ), on peut considérer qu'il existe d'autres paramètres qui peuvent influencer la production des ions bromate.

Sur la base des caractéristiques physicochimiques qui sont à notre disposition, nous avons essayé d'apporter quelques éléments de réflexion sur les écarts mis en évidence. 
Les données de la littérature ont montré que la présence d'azote ammoniacal $\mathrm{N}-\mathrm{NH}_{4}^{+}$limite la formation des ions bromate (GLAZE et al., 1993 ; DANIEL et al., 1993 ; KRASNER et al. 1993-b ainsi que LEGUBE et al., 1994). Toutes les eaux étudiées lors de ces campagnes ont présenté des teneurs inférieures à $0,01 \mathrm{mg} \mathrm{N} \cdot \mathrm{L}^{-1}$ (limite de détection de la méthode analytique), condition effaçant ainsi le rôle potentiel de ce paramètre.

De même, les écarts de $\mathrm{pH}$ sont trop faibles pour induire un effet significatif sur la production des ions bromate.

Notre réflexion s'est alors portée sur la matière organique dissoute (teneur et nature) ainsi que sur l'alcalinité des eaux.

Entre les eaux des campagnes 2 et 3 d'une part et celle de la campagne 6 d'autre part, on remarque qu'il existe des différences significatives au niveau de la teneur en COD et de l'alcalinité.

En effet, la pente de la droite $\left[\mathrm{BrO}_{3}\right]=f(\mathrm{C} \cdot \tau)$ est plus forte pour la campagne $\mathrm{n}^{\circ} 6$ dont le COD et l'alcalinité sont respectivement $3,7 \mathrm{mg} \cdot \mathrm{L}^{-1} \mathrm{COD}$ et 3,9 méq $\cdot \mathrm{L}^{-1}$ que celles obtenues pour les campagnes 2 et 3 qui présentent un COD de l'ordre de $2,6-2,7 \mathrm{mg} \cdot \mathrm{L}^{-1}$ et une alcalinité comprise entre 4,6 et 5,0 méq $\cdot L^{-1}$.

- En ce qui concerne la teneur en matière organique dissoute d'une part, si les travaux préliminaires réalisés par KOUDJONOU (1996) n'ont pas montré d'effet significatif de la variation de la concentration en COD sur la formation des ions bromate à C - $\tau$ constant, en revanche KRASNER et al. (1993) ont montré que pour un $\mathrm{C} \cdot \tau$ équivalent, plus le COD était grand, plus la formation d'ions bromate était importante.

Si la teneur en COD augmente, le maintien d'un C. $\tau$ constant nécessite la mise en œuvre d'un taux d'ozone appliqué plus élevé pour satisfaire la demande en ozone de la matière organique dissoute, conditions favorisant la production des ions bromate selon ces auteurs.

- Si on s'intéresse d'autre part à l'alcalinité, celle de l'eau de la campagne 6 étant plus faible que celles des campagnes 2 et 3 , le rôle " scavenger " des ions bicarbonate est par conséquent moins marqué. Selon vON GUNTEN and HOIGNÉ (1994), la formation d'ions bromate ferait largement intervenir les radicaux hydroxyles, condition de formation qui serait par conséquent favorisée dans le cas de l'eau de la campagne 6 . Toutefois, cette hypothèse ne prend pas en compte le rôle potentiel des radicaux bicarbonates et carbonates générés dans le milieu réactionnel, entités qui selon ces derniers auteurs, contribueraient également à l'accroissement de la production des ions bromate.

Les écarts relevés au niveau des campagnes 2 et 3 sont plus difficiles à discuter. On notera que la matière organique dissoute de l'eau de la campagne 3 est de nature sensiblement plus aromatique que celle de la campagne 2 si on se réfère au rapport UV/COD qui sont respectivement 2,15 et $1,88 \mathrm{~m}^{-1} \cdot \mathrm{L} \cdot \mathrm{mg}^{-1} \mathrm{C}$. La présence plus importante de sites aromatiques, fortement consommateurs d'ozone, pourrait être également un facteur à prendre en considération. 


\subsection{2 Étude des eaux issues de sites différents}

La figure 9 présente les résultats obtenus sur les eaux filtrées sable de Mérysur-Oise, Neuilly-sur-Marne et Choisy-le-Roi lors de la campagne 6.

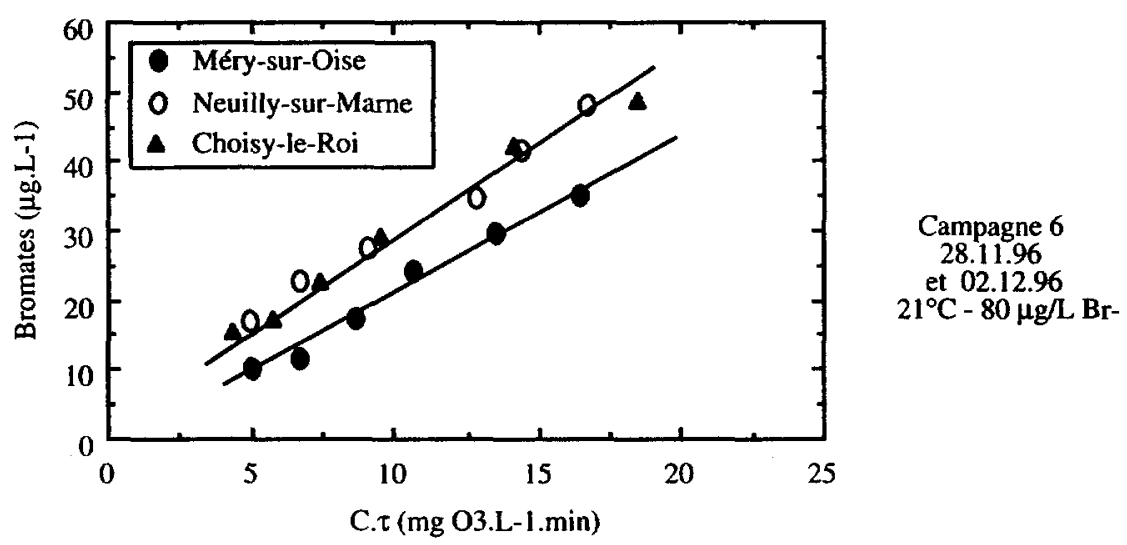

Figure 9 Formation d'ions bromate lors de l'ozonation d'eaux clarifiées d'origines diverses : étude comparative en laboratoire à température et teneur initiale en ions bromure constantes.

Bromate formation versus $C \cdot \tau$ : Importance of the origin of the clarified water at constant temperature and similar initial bromide content (lab-scale reactor).

Les résultats obtenus montrent qu'à température et teneur en ions bromure identiques, l'eau filtrée sable de Méry-sur-Oise s'est avérée le milieu le moins précurseur d'ions bromate, les eaux de Neuilly-sur-Marne et Choisy-le-Roi se comportant elles de façon similaire.

De nouveau, les rôles éventuels de l'azote ammoniacal et du pH ne peuvent pas être mis en cause pour ces deux campagnes.

Le paramètre qui présente les différences les plus marquées entre ces trois sites est le COD puisque l'eau filtrée sable de Méry-sur-Oise dont le COD a atteint $3,7 \mathrm{mg} \cdot \mathrm{L}^{-1}$ produit significativement moins d'ions bromate lors de l'ozonation, comparée aux deux autres eaux dont le COD est de l'ordre de 2,2 à $2,5 \mathrm{mg} \cdot \mathrm{L}^{-1}$.

Notons que ces résultats sont en contradiction avec ceux obtenus précédemment (figure 8) où l'augmentation du COD favorisait la formation des ions bromate. Dans le cas présent, l'augmentation du COD inhibe partiellement la formation des ions bromate.

Devant la divergence des résultats obtenus et par conséquent la grande difficulté de leur interprétation sur la base des mécanismes connus, le chercheur est en droit de s'interroger sur l'importance de la nature de la matière organique dissoute (aspect structural) dans la production des ions bromate. 


\section{4- CONCLUSION}

Tous les travaux conduits sur le pilote semi-industriel du site de Méry-sur-Oise et sur le module de laboratoire (eau filtrée sable) ont confirmé que la formation des ions bromate par ozonation était directement proportionnelle au C. $\tau$ appliqué (pour les valeurs de $\mathrm{C} \cdot \tau$ comprises entre 5 et $20 \mathrm{mg} \mathrm{O}_{3} \cdot \mathrm{L}^{-1} \cdot \mathrm{min}$ ) quelle que soit l'eau étudiée.

De plus, nous avons montré que le pilote de laboratoire permettait de reproduire de façon très satisfaisante les conditions d'ozonation du pilote de Méry-surOise. En effet, sur trois prélèvements d'eau effectués (eau filtrée sable de Mérysur-Oise) à des périodes différentes, les essais menés en parallèle sur le pilote semi-industriel et sur le pilote de laboratoire (dans les mêmes conditions de température) ont permis d'obtenir des relations $\left[\mathrm{BrO}_{3}\right]=f(\mathrm{C} \cdot \tau)$ comparables dans chaque cas.

La comparaison des résultats obtenus, quelle que soit l'eau étudiée (Mérysur-Oise, Neuilly-sur-Marne et Choisy-le-Roi), nous a permis d'extraire d'autres conclusions indiscutables comme :

- La formation des ions bromate augmente avec la température de l'eau (tous les autres paramètres étant constants).

- La formation des ions bromate augmente avec la concentration initiale en ions bromure (tous les autres paramètres étant constants).

II est cependant nouveau et intéressant de constater que même une très faible élévation de la concentration initiale en ions bromure $\left(+15 \mu \mathrm{g} \cdot \mathrm{L}^{-1}\right.$ obtenue par dopage à partir d'une solution concentrée de bromure de potassium KBr) a des effets non négligeables sur la formation des ions bromate. Par conséquent, les fluctuations naturelles ou accidentelles de plus de $20 \mu \mathrm{g} \cdot \mathrm{L}^{-1}$ de la teneur en ions bromure de l'eau d'alimentation entre l'hiver et l'été peuvent avoir un impact majeur sur la formation des ions bromate dans les filières de production d'eau potable.

Enfin, l'ensemble des mêmes résultats nous a permis d'interpréter d'autres phénomènes secondaires ayant une influence (moins certaine) sur la formation des ions bromate, comme :

- La formation des ions bromate augmente légèrement lorsque l'alcalinité diminue (tous les autres paramètres étant constants).

- La concentration et la nature (caractéristiques structurales) de la matière organique semblent jouer un rôle significatif dans le mécanisme de formation des ions bromate. Son intervention dans le cycle de décomposition radicalaire de l'ozone est une hypothèse à approfondir.

Globalement, cette étude tend à montrer que la production des ions bromate lors de la potabilisation d'eaux par l'ozone, dépendrait principalement des paramètres physico-chimiques de ces eaux (nature et concentration en matière organique naturelle, température, teneur en ions bromure, alcalinité...) que du type de réacteur utilisé. 


\section{REMERCIEMENTS}

Les auteurs remercient le Syndicat des Eaux d'lle-de-France (SEDIF) pour son soutien financier.

\section{RÉFÉRENCES BIBLIOGRAPHIQUES}

AMY G., SIDDIQUI M., OZEKIN K., ZHU H., WANG C., 1995. Empirically based models for predicting chlorination and ozonation by-products: haloacetic acids, chloral hydrate and bromate. Office of ground water and drinking water, US EPA $\mathrm{n}^{\circ} \mathrm{CX}$ 819579.

AMY G., WESTERHOFF P., MINEAR R., SONG R., 1997. Formation and control of brominated ozone byproducts. AWWA RF reports.

BADER H., HOIGNE, 1981. Determination of ozone in water by the indigo method. Wat. Res., 15, 449-456.

DANIEL P.A., ZAFER M.A., MEYERHOFER P.F., 1993. Bromate control : water quality, engineering and operational considerations. AIDE-IWSA, Paris, Nov. 22-24, Proceed. 181-188.

GLAZE W.H., WEINBERG H.S., CAVANAGH J.E., 1993. Evaluating the formation of brominated DBPs during ozonation. Journ. AWWA, 85, 96-103.

JOUANAL OFFICIEL DES COMMUNAUTÉS EUROPÉENNES, 1995. Proposition de directive du conseil relative à la qualité des eaux destinées à la consommation humaine, $n^{\circ} \mathrm{C} 131 / 5,31 / 05 / 95$.

KOUDJONOU B.K., 1996. Étude des conditions de formation des ions bromate lors de l'ozonation des eaux contenant des ions bromure. Th. Doct. Univ. Poitiers, France, $171 \mathrm{p}$.

KOUDJONOU B.K., MÜLLER M.C., COSTENTIN E., RACAUD P., VAN DER JAGT H., VILARO J.S., HUTCHINSON J., 1996. Bromate ion analysis by ion chromatography, Ozone Sci. \& Eng., 17, 561-573

KRASNER S.W., GRAMITH J.T., COFFEY B.M., YATES R.S., 1993-a. Impact of water quality and operational parameters on the formation and control of bromate during ozonation. AIDE-IWSA workshop, Paris-France, Nov. 22-24, 157-168.

KRASNER S.W., GLAZE W.H., WEINBERG H.S., DANIEL P.A., NAJM I., 1993b. Formation and control of bromate during ozonation of waters containing bromide. Journ. AWWA, 85, 73-80.

KRUITHOF J.C., ODERWALD-MULLER E.J., MEIJERS R.T, 1995. Control strategies for the restriction of bromate formation. 12th Ozone World Congress, 1OA, 15-18 May, Lille, France, Proceed. 209-221.

LEGUBE B., BOURBIGOT M.M., DEGUIN A., FIELDING M., KRUITOF J.C., MALLEVIALE J., MATIA L., MONTIEL A., 1994. Bromide/bromate survey on european water utilities. Proceed AWWA-WQTC, San Francisco 6-10 Nov, 965-978.

LEGUBE B., BOURBIGOT M.M., DEGUIN A., FIELDING M., KRUITOF J.C., MALLEVIALE J., MATIA L., MONTIEL A., WILBOURN, 1995. A survey of bromate in european drinking water. 12th Ozone World Congress, IOA, 15-18 May, Lille, France, 129-176.

ROUSTAN M., DUGET J.P., LAINE J.M., DOQUANG Z., MALLEVIALLE J., 1996. Bromate ion formation : Impact of ozone contactor hydraulics and operating conditions. Ozone Sci. \& Eng., 18, 87-97

SONG R., MINEAR R., WESTERHOFF P., AMY G., 1996. Bromate formation and control during water ozonation. Env. Technol., 17, 861-868

VON GUNTEN U., HOIGNÉ J., 1994. Bromate formation during ozonation of bromide containing waters: interaction of ozone and hydroxyl radical reactions. Environ. Sci. Technol., 28, 1234-1242 